ТTP97-32

hep-ph/9708387

August 1997

\title{
Current Issues in CP Violation ${ }^{1}$
}

\author{
Robert Fleischer $^{2}$ \\ Institut für Theoretische Teilchenphysik \\ Universität Karlsruhe \\ D-76128 Karlsruhe, Germany
}

\begin{abstract}
A brief review of $\mathrm{CP}$ violation in $K$ and $B$ decays is given. While the observables $\varepsilon$ and $\operatorname{Re}\left(\varepsilon^{\prime} / \varepsilon\right)$ describing CP violation in neutral $K$ decays do not allow a powerful test of the CKM mechanism of CP violation, rare $K \rightarrow \pi \nu \bar{\nu}$ decays and in particular the $B$-meson system are much more promising in this respect. After a brief look at the $K$ system, selected aspects of CP-violating effects in $B$ decays are discussed. It is pointed out that combined branching ratios for $B \rightarrow \pi K$ modes, which have been observed recently by the CLEO collaboration, may allow to derive stringent constraints on the CKM angle $\gamma$ that could open a window to new physics.
\end{abstract}

\section{INTRODUCTION}

The violation of the CP symmetry plays a central and fundamental role in modern particle physics. One of the reasons is that this phenomenon could guide us to physics beyond the Standard Model. To this end it is crucial to search for processes or relations among CP-violating observables that can be predicted in a clean way in the Standard Model framework and are not affected by hadronic uncertainties.

Within the Standard Model of electroweak interactions [1], CP violation is closely related to the Cabibbo-Kobayashi-Maskawa matrix (CKM matrix) $[2,3]$ connecting the electroweak eigenstates of the $d$-, $s$ - and $b$-quarks with their mass eigenstates. Whereas a single real parameter - the Cabibbo angle - is sufficient to parametrize the CKM matrix in the case of two fermion generations [2], three generalized Cabibbo-type angles and a single complex phase are needed in the three generation case [3]. This complex phase is the origin of $\mathrm{CP}$ violation within the Standard Model.

It turns out that $\mathrm{CP}$-violating observables are proportional to the following combination of CKM matrix elements:

1) Invited plenary talk given at Beyond the Standard Model $V$, Balholm, Norway, April 29

- May 4, 1997. To appear in the proceedings.

2) Address after September 1, 1997: Theory Division, CERN, CH-1211 Geneva 23, Switzerland 


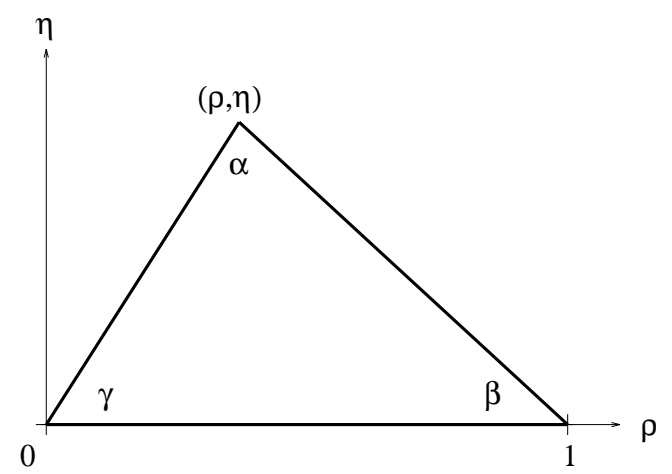

FIGURE 1. The unitarity triangle of the CKM matrix in the $(\rho, \eta)$ plane.

$$
J_{\mathrm{CP}}= \pm \operatorname{Im}\left(V_{i \alpha} V_{j \beta} V_{i \beta}^{*} V_{j \alpha}^{*}\right) \quad(i \neq j, \alpha \neq \beta),
$$

which represents a measure of the "strength" of CP violation within the Standard Model [4]. Since $J_{\mathrm{CP}}=\mathcal{O}\left(10^{-5}\right)$, CP violation is a small effect. In scenarios of new physics [5], typically several new complex couplings are present yielding additional sources of $\mathrm{CP}$ violation.

Concerning phenomenological applications, the parametrization

$$
\hat{V}_{\mathrm{CKM}}=\left(\begin{array}{ccc}
1-\frac{1}{2} \lambda^{2} & \lambda & A \lambda^{3} R_{b} e^{-i \gamma} \\
-\lambda & 1-\frac{1}{2} \lambda^{2} & A \lambda^{2} \\
A \lambda^{3} R_{t} e^{-i \beta} & -A \lambda^{2} & 1
\end{array}\right)+\mathcal{O}\left(\lambda^{4}\right)
$$

with $\lambda=0.22$ and

$$
\begin{aligned}
A & \equiv \frac{1}{\lambda^{2}}\left|V_{c b}\right|=0.81 \pm 0.06 \\
R_{b} & \equiv \frac{1}{\lambda}\left|\frac{V_{u b}}{V_{c b}}\right|=\sqrt{\rho^{2}+\eta^{2}}=0.36 \pm 0.08 \\
R_{t} & \equiv \frac{1}{\lambda}\left|\frac{V_{t d}}{V_{c b}}\right|=\sqrt{(1-\rho)^{2}+\eta^{2}}=\mathcal{O}(1)
\end{aligned}
$$

turns out to be very useful. This parametrization is a modification of the Wolfenstein parametrization [6] making not only the hierarchy of the CKM elements, but also the dependence on the angles $\beta=\beta(\rho, \eta)$ and $\gamma=\gamma(\rho, \eta)$ of the usual "non-squashed" unitarity triangle of the CKM matrix explicit [7]. This triangle is a graphical illustration of the fact that the CKM matrix is unitary and is sketched for completeness in Fig. 1.

\section{A BRIEF LOOK AT CP VIOLATION IN K DECAYS}

Although CP violation was discovered already in 1964 by observing $K_{\mathrm{L}} \rightarrow$ $\pi \pi$ decays [8], so far CP violation has been measured directly only within 
the neutral $K$-meson system, where it is described by two complex quantities called $\varepsilon$ and $\varepsilon^{\prime}$ which are defined by the following ratios of decay amplitudes:

$$
\frac{A\left(K_{\mathrm{L}} \rightarrow \pi^{+} \pi^{-}\right)}{A\left(K_{\mathrm{S}} \rightarrow \pi^{+} \pi^{-}\right)}=\varepsilon+\varepsilon^{\prime}, \quad \frac{A\left(K_{\mathrm{L}} \rightarrow \pi^{0} \pi^{0}\right)}{A\left(K_{\mathrm{S}} \rightarrow \pi^{0} \pi^{0}\right)}=\varepsilon-2 \varepsilon^{\prime} .
$$

While $\varepsilon=(2.26 \pm 0.02) \cdot e^{i \frac{\pi}{4}} \cdot 10^{-3}$ parametrizes "indirect" $\mathrm{CP}$ violation originating from the fact that the mass eigenstates of the neutral $K$-meson system are not eigenstates of the $\mathrm{CP}$ operator, the quantity $\operatorname{Re}\left(\varepsilon^{\prime} / \varepsilon\right)$ provides a measure of "direct" CP violation in $K \rightarrow \pi \pi$ transitions. The CP-violating observable $\varepsilon$ plays an important role to constrain the unitarity triangle [9] and informs us in particular about a non-vanishing, positive value of $\eta$.

Despite enormous efforts, the experimental situation concerning $\operatorname{Re}\left(\varepsilon^{\prime} / \varepsilon\right)$ is still unclear at present. Whereas the CERN experiment NA31 finds $\operatorname{Re}\left(\varepsilon^{\prime} / \varepsilon\right)=$ $(23 \pm 7) \cdot 10^{-4}$ indicating already direct $\mathrm{CP}$ violation, the result $\operatorname{Re}\left(\varepsilon^{\prime} / \varepsilon\right)=$ $(7.4 \pm 5.9) \cdot 10^{-4}$ of the Fermilab experiment E731 provides no unambiguous evidence for a non-zero effect. In the near future this situation will hopefully be clarified by improved measurements [10]. From a theoretical point of view, analyses of $\operatorname{Re}\left(\varepsilon^{\prime} / \varepsilon\right)$ are very involved and suffer at present from large hadronic uncertainties [9]. Consequently that observable does not allow a powerful test of the Standard Model description of CP violation unless the hadronic matrix elements of the relevant operators are under better control. The major goal of a possible future observation of $\operatorname{Re}\left(\varepsilon^{\prime} / \varepsilon\right) \neq 0$ would probably be the unambiguous exclusion of "superweak" models of CP violation [11].

More promising in respect of testing the CP-violating sector of the Standard Model are the rare decays $K_{\mathrm{L}} \rightarrow \pi^{0} \nu \bar{\nu}$ and $K^{+} \rightarrow \pi^{+} \nu \bar{\nu}$. These decays, in particular the first one, are very clean from a theoretical point of view [12]. Using the top-quark mass $m_{t}$ and the CKM element $\left|V_{c b}\right|$ as an additional input, the branching ratios of these decays allow a determination of the unitarity triangle. A detailed analysis shows that in particular $\sin (2 \beta)$ can be extracted with respectable accuracy [13]. Thus, comparing the value of $\sin (2 \beta)$ determined that way with the one extracted from $\mathrm{CP}$ violation in the "gold-plated" mode $B_{d} \rightarrow J / \psi K_{\mathrm{S}}$ (see the following section) one has a powerful tool to probe physics beyond the Standard Model. Unfortunately the branching ratios for $K_{\mathrm{L}} \rightarrow \pi^{0} \nu \bar{\nu}$ and $K^{+} \rightarrow \pi^{+} \nu \bar{\nu}$ are of $\mathcal{O}\left(10^{-11}\right)$ and $\mathcal{O}\left(10^{-10}\right)$, respectively, within the Standard Model [9] making measurements of these modes, especially of the former one, very challenging. Nevertheless there are plans to search for these important decays at BNL, FNAL and KEK.

At present the observed $\mathrm{CP}$ violation in the $K$ system can be described successfully by the Standard Model. This feature is, however, not surprising since so far only a single CP-violating observable, $\varepsilon$, has to be fitted. Consequently many different non-standard model descriptions of $\mathrm{CP}$ violation are imaginable [5]. From the brief discussion given above it is obvious that the $K$-meson system by itself cannot provide the whole picture of $\mathrm{CP}$ violation. 
Therefore it is essential to study $\mathrm{CP}$ violation outside this system. In this respect the $B$ system appears to be most promising which is also reflected by the tremendous experimental efforts at future $B$ factory facilities [14]. Let me note that there are also other interesting systems to investigate $\mathrm{CP}$ violation and to search for physics beyond the Standard Model, e.g. the $D$-meson system where sizable mixing or CP-violating effects would signal new physics because of the tiny Standard Model "background" [15]. Unfortunately I cannot discuss these systems in more detail in this presentation and shall focus on $B$ decays in the subsequent section.

\section{CP VIOLATION IN B DECAYS}

As far as CP violation and strategies for extracting angles of the unitarity triangle are concerned, the major role in the $B$ system is played by nonleptonic decays which can be divided into three decay classes: decays receiving both tree and penguin contributions, pure tree decays, and pure penguin decays. There are two types of penguin topologies: gluonic (QCD) and electroweak $(\mathrm{EW})$ penguins originating from strong and electroweak interactions, respectively. Interestingly also the latter operators play an important role in several processes because of the large top-quark mass [16].

In order to analyze non-leptonic $B$ decays theoretically, one uses low energy effective Hamiltonians that are calculated by making use of the operator product expansion yielding transition matrix elements of the structure

$$
\left\langle f\left|\mathcal{H}_{\mathrm{eff}}\right| i\right\rangle \propto \sum_{k} C_{k}(\mu)\left\langle f\left|Q_{k}(\mu)\right| i\right\rangle .
$$

The operator product expansion allows one to separate the short-distance contributions to Eq. (7) from the long-distance contributions described by perturbative Wilson coefficient functions $C_{k}(\mu)$ and non-perturbative hadronic matrix elements $\left\langle f\left|Q_{k}(\mu)\right| i\right\rangle$, respectively. As usual, $\mu$ denotes an appropriate renormalization scale. Examples for such Hamiltonians and a discussion of the technicalities arising in calculations of Wilson coefficients beyond the leading logarithmic approximation can be found in a recent review [17].

\section{CP Asymmetries in Neutral B Decays}

A particular simple and interesting situation arises if we restrict ourselves to decays of neutral $B_{q}$ mesons $(q \in\{d, s\})$ into CP self-conjugate final states $|f\rangle$ satisfying the relation $(\mathcal{C P})|f\rangle= \pm|f\rangle$. In that case the corresponding time-dependent $\mathrm{CP}$ asymmetry can be expressed as

$$
a_{\mathrm{CP}}(t) \equiv \frac{\Gamma\left(B_{q}^{0}(t) \rightarrow f\right)-\Gamma\left(\overline{B_{q}^{0}}(t) \rightarrow f\right)}{\Gamma\left(B_{q}^{0}(t) \rightarrow f\right)+\Gamma\left(\overline{B_{q}^{0}}(t) \rightarrow f\right)}=
$$




$$
\mathcal{A}_{\mathrm{CP}}^{\mathrm{dir}}\left(B_{q} \rightarrow f\right) \cos \left(\Delta M_{q} t\right)+\mathcal{A}_{\mathrm{CP}}^{\text {mix-ind }}\left(B_{q} \rightarrow f\right) \sin \left(\Delta M_{q} t\right),
$$

where the direct $\mathrm{CP}$-violating contributions have been separated from the mixing-induced CP-violating contributions which are characterized by

$$
\mathcal{A}_{\mathrm{CP}}^{\mathrm{dir}}\left(B_{q} \rightarrow f\right) \equiv \frac{1-\left|\xi_{f}^{(q)}\right|^{2}}{1+\left|\xi_{f}^{(q)}\right|^{2}} \quad \text { and } \quad \mathcal{A}_{\mathrm{CP}}^{\text {mix }} \text {-ind }\left(B_{q} \rightarrow f\right) \equiv \frac{2 \operatorname{Im} \xi_{f}^{(q)}}{1+\left|\xi_{f}^{(q)}\right|^{2}},
$$

respectively. Here direct $\mathrm{CP}$ violation refers to $\mathrm{CP}$-violating effects arising directly in the corresponding decay amplitudes, whereas mixing-induced $\mathrm{CP}$ violation is related to interference between $B_{q}^{0}-\overline{B_{q}^{0}}$ mixing and decay processes. Note that the expression Eq. (8) has to be modified in the $B_{s}$ case for $t \gtrsim$ $1 / \Delta \Gamma_{s}$ because of the expected sizable width difference $\Delta \Gamma_{s}[18]$.

In general the observable

$$
\xi_{f}^{(q)} \equiv e^{-i \phi_{\mathrm{M}}^{(q)}} \frac{A\left(\overline{B_{q}^{0}} \rightarrow f\right)}{A\left(B_{q} \rightarrow f\right)},
$$

where

$$
\phi_{\mathrm{M}}^{(q)}=\left\{\begin{array}{cc}
2 \beta & \text { for } q=d \\
0 & \text { for } q=s
\end{array}\right.
$$

denotes the weak $B_{q}^{0}-\overline{B_{q}^{0}}$ mixing phase, suffers from large hardonic uncertainties that are introduced through the decay amplitudes $A$. There is, however, a very important special case where these uncertainties cancel. It is given if $B_{q} \rightarrow f$ is dominated by a single CKM amplitude. In that case $\xi_{f}^{(q)}$ takes the simple form

$$
\xi_{f}^{(q)}=\mp \exp \left[-i\left(\phi_{\mathrm{M}}^{(q)}-\phi_{\mathrm{D}}^{(f)}\right)\right],
$$

where $\phi_{\mathrm{D}}^{(f)}$ is a characteristic weak decay phase that is given by

$$
\phi_{\mathrm{D}}^{(f)}=\left\{\begin{array}{cl}
-2 \gamma & \text { for dominant } \bar{b} \rightarrow \bar{u} u \bar{r} \text { CKM amplitudes in } B_{q} \rightarrow f \\
0 & \text { for dominant } \bar{b} \rightarrow \bar{c} c \bar{r} \text { CKM amplitudes in } B_{q} \rightarrow f .
\end{array}\right.
$$

Here the label $r \in\{d, s\}$ distinguishes between $b \rightarrow d$ and $b \rightarrow s$ transitions.

The most important application of this formalism is the "gold-plated" decay $B_{d} \rightarrow J / \psi K_{\mathrm{S}}$ [19]. If one goes through the relevant Feynman diagrams contributing to this channel one finds that it is dominated to excellent accuracy by the $\bar{b} \rightarrow \bar{c} c \bar{s}$ CKM amplitude since penguins enter essentially with the same weak phase as the leading tree contribution (see e.g. [16] for a recent detailed discussion). Therefore the weak decay phase vanishes and we get

$$
\mathcal{A}_{\mathrm{CP}}^{\text {mix-ind }}\left(B_{d} \rightarrow J / \psi K_{\mathrm{S}}\right)=+\sin [-(2 \beta-0)] .
$$


This CP-violating observable allows hence a clean determination of the angle $\beta$ of the unitarity triangle (up to discrete ambiguities; see [20] for a recent discussion) and is also very promising from an experimental point of view for future $B$ factories [14].

Another important decay is $B_{d} \rightarrow \pi^{+} \pi^{-}$which would measure $-\sin (2 \alpha)$ in a clean way through

$$
\mathcal{A}_{\mathrm{CP}}^{\mathrm{mix}-\mathrm{ind}}\left(B_{d} \rightarrow \pi^{+} \pi^{-}\right)=-\sin [-(2 \beta+2 \gamma)]=-\sin (2 \alpha)
$$

if there were no penguins present. However, penguins do contribute to $B_{d} \rightarrow \pi^{+} \pi^{-}$. The corresponding hadronic uncertainties affecting the determination of $\alpha$ were discussed by many authors in the previous literature. There are even methods to control these uncertainties in a quantitative way. Unfortunately these strategies are usually rather challenging in practice. The most important examples are the $B \rightarrow \pi \pi$ isospin triangles proposed by Gronau and London [21], and the approach using $B \rightarrow \rho \pi$ modes suggested by Snyder and Quinn [22]. An approximate method to correct for the penguin uncertainties in $B_{d} \rightarrow \pi^{+} \pi^{-}$that appears to be promising for the early days of the $B$ factory era was proposed in [23]. For a detailed discussion of these and other strategies the reader is referred to reviews on this subject, e.g. [16,24].

A decay appearing frequently as a tool to determine the angle $\gamma$ of the unitarity triangle is $B_{s} \rightarrow \rho^{0} K_{\mathrm{S}}$. There, however, penguins are expected to lead to serious problems - more serious than in $B_{d} \rightarrow \pi^{+} \pi^{-}$- so that this mode appears to be the "wrong" way to extract $\gamma[16]$. Other strategies allowing meaningful determinations of this angle will be discussed in a moment.

\section{CP Violation in Non-leptonic Penguin Modes}

In view of testing the Standard Model description of $\mathrm{CP}$ violation, penguininduced modes play an important role. Because of the loop-suppression of these "rare" FCNC processes, it is plausible - and indeed the case in specific model calculations - that new physics contributions to these decays are of similar magnitude as those of the Standard Model [5]. An example is the $b \rightarrow d$ penguin mode $B_{d} \rightarrow K^{0} \overline{K^{0}}$ (see e.g. [25] for an analysis of new physics effects). If one assumes that penguins with internal top-quarks play the dominant role in this decay, the weak $B_{d}^{0}-\overline{B_{d}^{0}}$ mixing and $B_{d} \rightarrow K^{0} \overline{K^{0}}$ decay phases cancel in the corresponding observable $\xi_{K^{0}}^{(d)} \overline{K^{0}}$ implying vanishing $\mathrm{CP}$ violation in that decay. Consequently one would conclude that a measurement of non-vanishing CP violation in $B_{d} \rightarrow K^{0} \overline{K^{0}}$ would signal physics beyond the Standard Model. However, long-distance effects related to penguins with internal charm- and up-quarks may easily spoil the assumption of top-quark dominance $[16,26]$. These contributions may lead to sizable CP violation in $B_{d} \rightarrow K^{0} \overline{K^{0}}$ even within the Standard Model [27], so that a measurement 
of such CP asymmetries would not necessarily imply new physics as claimed in several previous papers. Unfortunately a measurement of these effects will be very difficult since the Standard Model expectation for the corresponding branching ratio is $\mathcal{O}\left(10^{-6}\right)$ which is still one order of magnitude below the recent CLEO bound $\mathrm{BR}\left(B_{d} \rightarrow K^{0} \overline{K^{0}}\right)<1.7 \cdot 10^{-5}$ [28].

More promising in this respect and - more importantly - to search for physics beyond the Standard Model is the $b \rightarrow s$ penguin mode $B_{d} \rightarrow \phi K_{\mathrm{S}}$. The branching ratio for this decay is expected to be of $\mathcal{O}\left(10^{-5}\right)$ and may be large enough to investigate this channel at future $B$ factories. Interestingly there is to very good approximation no non-trivial CKM phase present in the corresponding decay amplitude [16], so that direct $\mathrm{CP}$ violation vanishes and mixing-induced $\mathrm{CP}$ violation measures simply the weak $B_{d}^{0}-\overline{B_{d}^{0}}$ mixing phase which is related to the angle $\beta$ of the unitarity triangle. It should be stressed that this statement does not require the questionable assumption of top-quark dominance in penguin amplitudes. Consequently an important probe for new physics in $b \rightarrow s$ FCNC processes is provided by the relation

$$
\mathcal{A}_{\mathrm{CP}}^{\text {mix-ind }}\left(B_{d} \rightarrow J / \psi K_{\mathrm{S}}\right)=\mathcal{A}_{\mathrm{CP}}^{\text {mix-ind }}\left(B_{d} \rightarrow \phi K_{\mathrm{S}}\right)=-\sin (2 \beta),
$$

which holds within the Standard Model framework. The theoretical accuracy of this relation is limited by certain neglected terms that are CKM-suppressed by $\mathcal{O}\left(\lambda^{2}\right)$ and may lead to tiny direct CP-violating asymmetries in $B_{d} \rightarrow \phi K_{\mathrm{S}}$ of at most $\mathcal{O}(1 \%)$ [16]. Recently the importance of $B_{d} \rightarrow \phi K_{\mathrm{S}}$ and similar modes like e.g. $B_{d} \rightarrow \eta^{\prime} K_{\mathrm{S}}$ to search for new physics in $b \rightarrow s$ transitions has been emphasized by several authors $[16,29]$.

\section{A Closer Look at the $B_{s}$ System}

In the $B_{s}$ system very rapid $B_{s}^{0}-\overline{B_{s}^{0}}$ oscillations are expected requiring an excellent vertex resolution system. Therefore studies of $\mathrm{CP}$ violation in $B_{s}$ decays are regarded as being very difficult. An alternative route to investigate CPviolating effects may be provided by the width difference $\Delta \Gamma_{s} / \Gamma_{s}=\mathcal{O}(20 \%)$ arising from CKM favoured $b \rightarrow c \bar{c} s$ transitions into final states that are common both to $B_{s}^{0}$ and $\overline{B_{s}^{0}}$. Because of this width difference already untagged data samples of $B_{s}$ decays may exhibit CP-violating effects [18].

Recently several "untagged" strategies to extract the CKM angle $\gamma$ were proposed using e.g. $B_{s} \rightarrow K^{+} K^{-}, K^{0} \overline{K^{0}}$ and $S U(3)$ flavor symmetry, angular distributions in $B_{s} \rightarrow K^{*+} K^{*-}, K^{* 0} \overline{K^{* 0}}$ and $S U(2)$ isospin symmetry, or angular distributions in $B_{s} \rightarrow D^{*} \phi, D_{s}^{* \pm} K^{* \mp}$ allowing a clean determination of $\gamma$ [30]. Compared to the tagged case, such untagged measurements are obviously much more promising in view of efficiency, acceptance and purity. A lot of statistics is required, however, and the natural place for these experiments seems to be a hadron machine. The feasibility of untagged strategies to extract CKM phases depends crucially on a sizable width difference $\Delta \Gamma_{s}$ and 
it is not yet clear whether it will turn out to be large enough to make these studies possible.

The $B_{s}$ system provides also an important probe for physics beyond the Standard Model through the decays $B_{s} \rightarrow D_{s}^{*+} D_{s}^{*-}$ and $B_{s} \rightarrow J / \psi \phi$, which is the counterpart of the "gold-plated" mode $B_{d} \rightarrow J / \psi K_{\mathrm{S}}$ to measure $\beta$. These modes are dominated by a single CKM amplitude and allow - in principal even from their untagged data samples [30] - the extraction of a CP-violating weak phase $\phi_{\mathrm{CKM}} \equiv 2 \lambda^{2} \eta$ which is expected to be of $\mathcal{O}(0.03)$, i.e. very small, within the Standard Model [9]. Consequently an extracted value of $\phi_{\text {CKM }}$ that is much larger than this Standard Model expectation would signal new physics. Similarly as in the case of the penguin modes discussed above, it is plausible that physics beyond the Standard Model could also play an important role in the loop-suppressed $B_{q}^{0}-\overline{B_{q}^{0}}$ mixing processes [5]. In the case of the $B_{s}$ system, a sizable mixing phase could originate from new physics leading e.g. to significant $\mathrm{CP}$ violation in $B_{s} \rightarrow J / \psi \phi$.

\section{Extracting CKM Angles with Amplitude Relations}

Since mixing effects are absent in the charged $B$ system, the measurement of a non-vanishing $\mathrm{CP}$ asymmetry in a charged $B$ decay would give unambiguous evidence for direct CP violation thereby ruling out "superweak" models. Such CP asymmtries arise from interference between decay amplitudes with both different $\mathrm{CP}$-violating weak and $\mathrm{CP}$-conserving strong phases. Whereas the weak phases are related to the CKM matrix, the strong phases are induced by strong final state interaction effects and introduce severe theoretical uncertainties into the calculation destroying in general the clean relation of the $\mathrm{CP}$ asymmetry to the phases of the CKM matrix.

Nevertheless there are decays of charged $B$ mesons which play an important role to extract angles of the unitarity triangle, in particular for $\gamma$. To this end amplitude relations - either exact or approximate ones based on flavor symmetries - are used. A recent review of these methods can be found in [16]. The "prototype" is the approach to determine $\gamma$ with the help of triangle relations among $B^{ \pm} \rightarrow D K^{ \pm}$decay amplitudes proposed by Gronau and Wyler [31]. Unfortunately the corresponding triangles are expected to be very "squashed" ones. Moreover one has to deal with additional experimental problems [32], so that this approach is very difficult from a practical point of view. Recently more refined variants have been proposed by Atwood, Dunietz and Soni [32].

About three years ago, several methods to extract CKM angles were presented by Gronau, Hernández, London and Rosner who have combined the $S U(3)$ flavor symmetry of strong interactions with certain dynamical assumptions to derive relations among $B \rightarrow \pi \pi, \pi K, K \bar{K}$ decay amplitudes [33]. This approach has been very popular over recent years and requires only a measurement of the relevant branching ratios. A closer look shows, however, that 
it suffers despite its attractiveness from several problems: the $S U(3)$ relations are not valid exactly, QCD penguins with internal charm- and up-quarks play in certain cases an important role, and interestingly also EW penguins lead to complications. In order to eliminate the EW penguin contributions, usually very involved strategies are needed. A detailed discussion of all these methods (mainly to extract $\gamma$ ) is beyond the scope of this article and the reader is referred to a recent review [16] and references therein.

\section{Searching for $\gamma$ and New Physics with $B \rightarrow \pi K$ Modes}

A simple approach to determine $\gamma$ with the help of the branching ratios for $B^{+} \rightarrow \pi^{+} K^{0}, B_{d}^{0} \rightarrow \pi^{-} K^{+}$and their charge conjugates was proposed in [34] (see also [16]). It makes use of the fact that the general phase structure of the corresponding decay amplitudes is known reliably within the Standard Model, and employs the $S U(2)$ isospin symmetry of strong interactions to relate the QCD penguin contributions. If the magnitude of the current-current amplitude $T^{\prime}$ contributing to $B_{d}^{0} \rightarrow \pi^{-} K^{+}$is known - it can be fixed e.g. through $B^{+} \rightarrow \pi^{+} \pi^{0}$, "factorization", or hopefully lattice gauge theory one day - two amplitude triangles can be constructed allowing in particular the extraction of $\gamma$. This approach is promising for future $B$ physics experiments, since it requires only time-independent measurements of branching ratios at the $\mathcal{O}\left(10^{-5}\right)$ level. If one measures in addition the branching ratios for $B^{+} \rightarrow$ $\pi^{0} K^{+}$and its charge-conjugate, also the $b \rightarrow s$ EW penguin amplitude can be determined which is an interesting probe for new physics [35].

Recently the CLEO collaboration has reported the first observation of the decays $B^{+} \rightarrow \pi^{+} K^{0}$ and $B_{d}^{0} \rightarrow \pi^{-} K^{+}$[28]. At present, however, only combined branching ratios, i.e. averaged ones over decays and their chargeconjugates, are available with large experimental uncertainties. Therefore it is unfortunately not yet possible to extract $\gamma$ from the triangle construction proposed in [34]. The recent CLEO measurements allow, however, to derive interesting constraints on $\gamma$ which are of the form

$$
0^{\circ} \leq \gamma \leq \gamma_{0} \quad \vee \quad 180^{\circ}-\gamma_{0} \leq \gamma \leq 180^{\circ}
$$

and are hence complementary to the presently allowed range

$$
42^{\circ} \lesssim \gamma \lesssim 135^{\circ}
$$

for that angle arising from the usual fits of the unitarity triangle [9]. This remarkable feature has been pointed out recently by Mannel and myself [36]. The quantity $\gamma_{0}$ in Eq. (17) depends both on the ratio

$$
R \equiv \frac{\mathrm{BR}\left(B_{d} \rightarrow \pi^{\mp} K^{ \pm}\right)}{\mathrm{BR}\left(B^{ \pm} \rightarrow \pi^{ \pm} K\right)}=\frac{\mathrm{BR}\left(B_{d}^{0} \rightarrow \pi^{-} K^{+}\right)+\mathrm{BR}\left(\overline{B_{d}^{0}} \rightarrow \pi^{+} K^{-}\right)}{\mathrm{BR}\left(B^{+} \rightarrow \pi^{+} K^{0}\right)+\mathrm{BR}\left(B^{-} \rightarrow \pi^{-} \overline{K^{0}}\right)}
$$




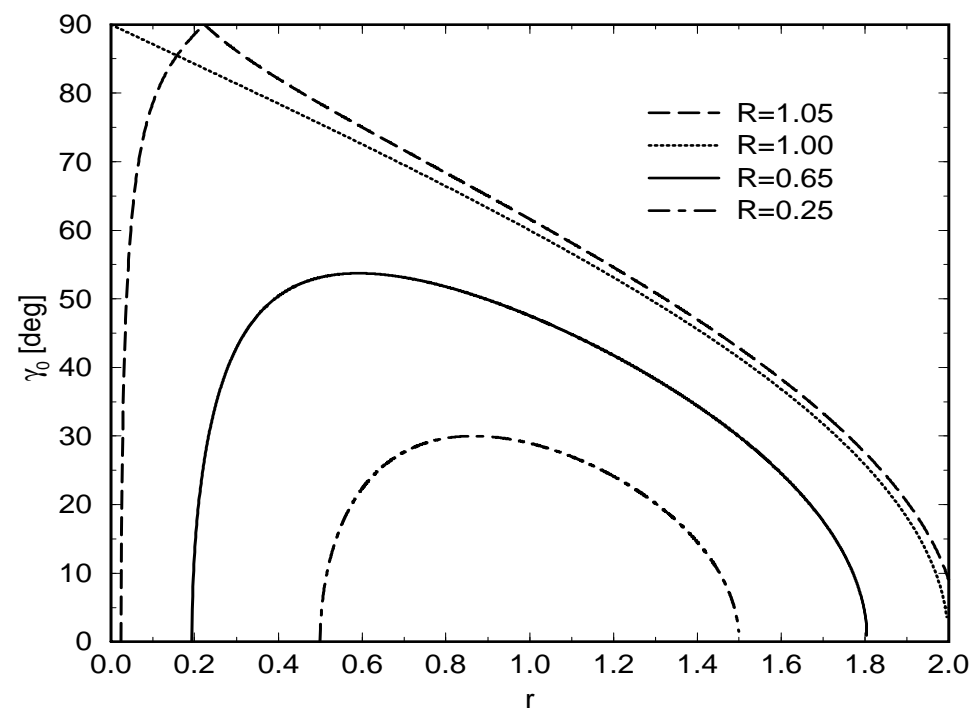

FIGURE 2. The dependence of $\gamma_{0}$ on the amplitude ratio $r$ for various values of $R$. The range of $R$ corresponds to recent CLEO measurements.

of the combined branching ratios and on the amplitude ratio

$$
r \equiv\left|T^{\prime}\right| /\left|P^{\prime}\right|
$$

of the current-current and penguin operator contributions to $B_{d} \rightarrow \pi^{\mp} K^{ \pm}$as can be seen in Fig. 2. If we look at that figure we observe that $R=1$ is a very important special case. For $R>1$ constraints on $\gamma$ require some knowledge about $r$, i.e. $\left|T^{\prime}\right|$, e.g. from $B^{+} \rightarrow \pi^{+} \pi^{0}$, "factorization", or hopefully lattice gauge theory one day. On the other hand, if $R$ is found experimentally to be smaller than one, bounds on $\gamma$ can always be obtained independent of $r$. The point is that $\gamma_{0}$ takes a maximal value

$$
\gamma_{0}^{\max }=\arccos (\sqrt{1-R})
$$

depending only on the ratio $R$ of combined $B \rightarrow \pi K$ branching ratios [36].

Let us take as an example the central values of the recent CLEO measurements [28] yielding $R=0.65$. This value corresponds to $\gamma_{0}^{\max }=54^{\circ}$ and implies the range $0^{\circ} \leq \gamma \leq 54^{\circ} \vee 126^{\circ} \leq \gamma \leq 180^{\circ}$ which has only the small overlap $42^{\circ} \lesssim \gamma \leq 54^{\circ} \vee 126^{\circ} \leq \gamma \lesssim 135^{\circ}$ with the range (18). The two pieces of this range are distinguished by the sign of a quantity $\cos \delta$, where $\delta$ is the $\mathrm{CP}$-conserving strong phase shift between the $T^{\prime}$ and $P^{\prime}$ amplitudes. Using arguments based on "factorization" one expects $\cos \delta>0$ corresponding to the former interval of that range, i.e. $42^{\circ} \lesssim \gamma \leq 54^{\circ}$ in our example [36] (see [37] for a recent model calculation). Consequently, once more data come in 
confirming $R<1$, the decays $B_{d} \rightarrow \pi^{\mp} K^{ \pm}$and $B^{ \pm} \rightarrow \pi^{ \pm} K$ may put the Standard Model to a decisive test and could open a window to new physics. The implications of physics beyond the Standard Model on the $B \rightarrow \pi K$ modes have been analyzed in a recent paper [38]. A striking new physics effect would e.g. be sizable $\mathrm{CP}$ violation in the decay $B^{+} \rightarrow \pi^{+} K^{0}$.

Let me finally note that the consistent description of $B^{ \pm} \rightarrow \pi^{ \pm} K$ and $B_{d} \rightarrow \pi^{\mp} K^{ \pm}$within the Standard Model implies in addition to the constraints on $\gamma$ discussed above the range

$$
|1-\sqrt{R}| \leq r \leq 1+\sqrt{R}
$$

for the amplitude ratio $r$ and upper limits for the CP-violating asymmetry in $B_{d}^{0} \rightarrow \pi^{-} K^{+}$. It is interesting to note that commonly accepted means to estimate $r$ yield values that are at the edge of compatibility with the present CLEO measurements [36].

In conclusion, I hope that the aspects of $\mathrm{CP}$ violation in $K$ and $B$ decays that I have selected for this presentation have convinced the reader that this phenomenon provides powerful tools to probe new physics. More advanced experimental studies of CP-violating effects in the Kaon system and the exploration of $\mathrm{CP}$ violation at $B$ physics facilities are just ahead of us. In the foreseeable future these experiments may bring unexpected results that could shed light on physics beyond the Standard Model. Certainly the coming years will be very exciting!

\section{ACKNOWLEDGMENTS}

I am very grateful to Gerald Eigen and Per Osland for inviting me to that most enjoyable conference and for providing generous travel support.

\section{REFERENCES}

1. S.L. Glashow, Nucl. Phys. 22, 579 (1961); S. Weinberg, Phys. Rev. Lett. 19, 1264 (1967); A. Salam, in Elementary Particle Theory, ed. N. Svartholm (Almqvist and Wiksell, Stockholm, 1968).

2. N. Cabibbo, Phys. Rev. Lett. 10, 531 (1963).

3. M. Kobayashi and T. Maskawa, Progr. Theor. Phys. 49, 652 (1973).

4. C. Jarlskog, Phys. Rev. Lett. 55, 1039 (1985); Z. Phys. C29, 491 (1985).

5. For reviews see e.g. Y. Grossman, Y. Nir and R. Rattazzi, hep-ph/9701231; M. Gronau and D. London, Phys. Rev. D55, 2845 (1997); Y. Nir and H.R. Quinn, Ann. Rev. Nucl. Part. Sci. 42, 211 (1992).

6. L. Wolfenstein, Phys. Rev. Lett. 51, 1945 (1983).

7. L.L. Chau and W.-Y. Keung, Phys. Rev. Lett. 53, 1802 (1984); C. Jarlskog and R. Stora, Phys. Lett. B208, 268 (1988). 
8. J.H. Christenson, J.W. Cronin, V.L. Fitch and R. Turlay, Phys. Rev. Lett. 13, 138 (1964).

9. For a review see A.J. Buras and R. Fleischer, hep-ph/9704376.

10. H. Nguyen, these proceedings.

11. L. Wolfenstein, Phys. Rev. Lett. 13, 562 (1964).

12. G. Buchalla and A.J. Buras, Nucl. Phys. B412, 106 (1994); Nucl. Phys. B400, 225 (1993).

13. G. Buchalla and A.J. Buras, Phys. Rev. D54, 6782 (1996); Phys. Lett. B333, 221 (1994); A.J. Buras, Phys. Lett. B333, 476 (1994).

14. D. Hitlin, these proceedings.

15. For a recent review see E. Golowich, hep-ph/9706548.

16. For a review see R. Fleischer, Int. J. Mod. Phys. A12, 2459 (1997).

17. G. Buchalla, A.J. Buras and M.E. Lautenbacher, Rev. Mod. Phys. 68, 1125 (1996).

18. I. Dunietz, Phys. Rev. D52, 3048 (1995).

19. A.B. Carter and A.I. Sanda, Phys. Rev. Lett. 45, 952 (1980); Phys. Rev. D23, 1567 (1981); I.I. Bigi and A.I. Sanda, Nucl. Phys. B193, 85 (1981).

20. Y. Grossman and H.R. Quinn, hep-ph/9705356.

21. M. Gronau and D. London, Phys. Rev. Lett. 65, 3381 (1990).

22. A. Snyder and H.R. Quinn, Phys. Rev. D48, 2139 (1993).

23. R. Fleischer and T. Mannel, Phys. Lett. B397, 269 (1997).

24. For reviews see e.g. Y. Nir and H.R. Quinn, in B Decays, ed. S. Stone (World Scientific, Singapore, 1994), p. 362; I. Dunietz, ibid., p. 393; H.R. Quinn, Nucl. Phys. B (Proc. Suppl.) 50, 17 (1996); M. Gronau, hep-ph/9611255; R. Fleischer, Ref. [16].

25. M.P. Worah, Phys. Rev. D54, 2198 (1996).

26. A.J. Buras and R. Fleischer, Phys. Lett. B341, 379 (1995). Recently this point has also been raised by M. Ciuchini et al., hep-ph/9703353.

27. R. Fleischer, Phys. Lett. B341, 205 (1994).

28. J.P. Alexander and B.H. Behrends, Proceedings of the 2nd International Conference on $B$ Physics and CP Violation, Honolulu, Hawaii, March 1997; F. Würthwein, hep-ex/9706010; K. Ecklund, these proceedings.

29. Y. Grossman and M.P. Worah, Phys. Lett. B395, 241 (1997); M. Ciuchini et al., hep-ph/9704274; D. London and A. Soni, hep-ph/9704277; R. Barbieri and A. Strumia, hep-ph/9704402.

30. R. Fleischer and I. Dunietz, Phys. Lett. B387, 361 (1996); Phys. Rev. D55, 259 (1997); R. Fleischer, hep-ph/9705404.

31. M. Gronau and D. Wyler, Phys. Lett. B265, 172 (1991).

32. D. Atwood, I. Dunietz and A. Soni, Phys. Rev. Lett. 78, 3257 (1997).

33. M. Gronau et al., Phys. Rev. D52, 6374 (1995) and references therein.

34. R. Fleischer, Phys. Lett. B365, 399 (1996).

35. A.J. Buras and R. Fleischer, Phys. Lett. B365, 390 (1996).

36. R. Fleischer and T. Mannel, hep-ph/9704423.

37. A. Ali and C. Greub, hep-ph/9707251.

38. R. Fleischer and T. Mannel, hep-ph/9706261. 